\title{
Low loss transmission in negative curvature optical fibers with elliptical capillary tubes
}

\author{
Subhasis Chaudhuri, Lieke D. Van Putten, Francesco Poletti, Pier J. A. Sazio
}

\begin{abstract}
Regular and nested negative curvature optical fibers with elliptical capillary tubes have been proposed in this paper for ultra-low loss guidance at the telecommunication wavelength. Finite element modeling shows that these fibers can have lower loss compared to their counterparts with circular tubes. These hollow core fibers with low loss and large bandwidth can have huge potential applications in data transmission, sensing, high power delivery and serve as an ideal platform for the study of a wide variety of nonlinear optical effects.
\end{abstract}

Index Terms - Antiresonant reflection, Hollow core optical fiber, Negative curvature fiber, Numerical modeling.

\section{INTRODUCTION}

$\mathrm{H}$ OLLOW core optical fibers have been widely studied in the past few decades [1,2]. Guiding light in hollow core ensures low absorption loss, low latency, absence of optical nonlinearities and no Fresnel reflections from the ends. Additionally the hollow core can serve as a cavity for the generation of X-Rays [3], the guiding of atoms and other mesoscale particles with laser light $[4,5]$, as well as the demonstration of nonlinear optical phenomena in liquids and gases. Several types of hollow core fibers exist such as the photonic bandgap fiber [1], hollow metallic waveguide [6], metal-dielectric fiber [7], antiresonant optical fiber [8-10], Bragg fiber $[11,12]$ etc. which guide light by a variety of different mechanisms. A wide variety of hollow core fibers have emerged in recent years whose intricate cladding designs enable antiresonant reflection [13]. Although it is well established that optical fibers that guide light by photonic bandgap formation have the lowest loss, a new class of antiresonant optical fiber, the negative curvature fiber has shown the potential of guiding light at even lower loss [14, 15]. A common type of negative curvature fiber consists of a hollow core surrounded by a row of circular capillary tubes that are attached to the cladding. These capillary tubes act as resonators which can decrease or increase the confinement of light in the air core under different resonant or antiresonant conditions. In such fibers with tube thickness $t$ and refractive index $n$, the low loss regions lie between the resonant wavelengths which are given by,

This work was supported by DARPA PULSE (1550650, MOD5). S. Chaudhuri is with the Department of Chemistry, The Pennsylvania State University, University Park, PA 16802 USA (e-mail: sqc5288@psu.edu).

$$
\lambda_{m} \approx \frac{2 t \sqrt{n^{2}-1}}{m} \text { where, } \mathrm{m}=1,2, \ldots \text { etc. }
$$

At these resonant wavelengths there is phase matching between air and glass modes in the tubes which lead to a high confinement loss. The negative curvature of the core formed due to the presence of the capillary tubes enhances the antiresonant effect which results in its low loss guidance. A number of designs for low loss guidance of such fibers have been proposed. Negative curvature fiber that guides light with a loss of $50 \mathrm{~dB} / \mathrm{km}$ at $3.39 \mu \mathrm{m}$ (core diameter $119 \mu \mathrm{m}$ ) [17] and $100 \mathrm{~dB} / \mathrm{km}$ at $3.1 \mu \mathrm{m}$ (core diameter $109 \mu \mathrm{m}$ ) have been demonstrated [18]. Different modifications of the negative curvature fiber have been proposed to further decrease the loss, such as adding another layer of tubes inside these capillary tubes thereby making nested fibers [16, 19]. Belardi demonstrated the guidance of $0.48 \mu \mathrm{m}$ wavelength in a nested fiber (core diameter $51 \mu \mathrm{m}$ ) with an attenuation of $175 \mathrm{~dB} / \mathrm{km}$ [20]. Further reduction in loss values can be expected with improvements in drawing techniques as well as with modifications in the structures of such fibers. In this paper we demonstrate via finite element modeling using COMSOL Multiphysics ${ }^{\circledR}$ software that the optical loss of different types of negative curvature fibers can decrease significantly by replacing the circular capillary tubes with elliptical ones. It has been proposed recently that this design can decrease the loss of these fibers at a wavelength of $1.06 \mu \mathrm{m}$ [21, 22]. In this paper we present a comprehensive analysis of designing low loss negative curvature fibers with elliptical tubes at the telecommunication wavelength of $1.55 \mu \mathrm{m}$. We have also shown for the first time that the loss can decrease even further if elliptical tubes are used for the designing of nested negative curvature fibers. Such improvements in design will enable the fabrication of the next generation of negative curvature fibers with lower loss that will open up new possibilities.

\section{NUMERICAL ANALYSIS OF NEGATIVE CURVATURE OPTICAL FIBERS}

The loss in a hollow core antiresonant optical fiber depends on the curvature of the cladding elements which in turn determine the effective curvature of the core. It has been shown that the loss in a Kagome fiber with negative curvature core

L. D. Van Putten, F. Poletti, P. J. A. Sazio are with the Optoelectronics Research Centre, University of Southampton, Southampton, SO17 1BJ, U.K. 
boundary decreases with the increase in the curvature of the cladding elements [23]. This is on account of the fact that on increasing the curvature, the coupling between core and cladding modes decreases enabling more power to be confined in the core which results in an overall decrease in loss [24]. Similar effect has been observed in hollow core fibers with "icecream cone" cladding elements as well [25]. Thus increasing the curvature of the cladding elements in fibers with negative curvature core results in overall decrease of loss. One way of achieving this is to alter the sizes of the circular cladding tubes in a negative curvature fiber. This impacts the effective curvature and thereby changes the overall guidance properties [25]. However, this change can be more pronounced if the circular tubes are replaced by elliptical ones. The major and minor axes (we define major axis as the axis that points radially towards the center of the fiber and the minor axis is perpendicular to it as shown in Fig. 1) of an ellipse can be tuned to change its curvature thereby having a more significant effect on the guidance properties which can lead to a lower loss than that attained by circular tubes.
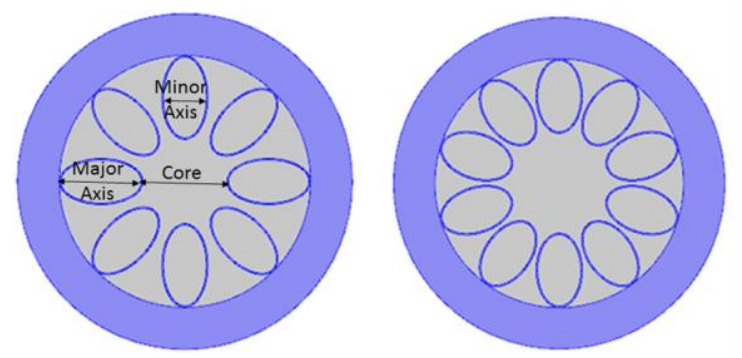

Fig. 1. Illustration of negative curvature fibers with elliptical tubes ( 8 and 10 tubes respectively). Blue regions are silica and the grey regions are air.
Using COMSOL modeling perfectly-matched layer boundary conditions are met to calculate the confinement loss of the $\mathrm{HE}_{11}$ mode accurately. Elliptical tubes have more parameters that can be varied compared to circular tubes, by using different values for the major and minor-axes. Other parameters that can be varied to optimize the design of the structure are the core size, the number of ellipses and the tube thickness. In this study these parameters are chosen to obtain antiresonance and thus low loss at $1.55 \mu \mathrm{m}$. Both the core diameter and the major axes of the ellipses have been fixed at $30 \mu \mathrm{m}$ while the other parameters have been varied. The number of ellipses that can be accommodated in the fiber can be increased by decreasing the minor axes i.e. making them slimmer. Fig. 2a shows the effect of varying the size of minor axes on the loss at a wavelength of $1.55 \mu \mathrm{m}$. Although, equation 1 primarily determines the antiresonance window that leads to low loss in a fiber with particular tube thickness, the effective curvature of the core has a profound impact on determining that loss. Thus, the sizes, shapes, number and area occupied by the cladding elements (these determine the effective curvature of the core) and the antiresonant conditions together determine what value of tube thickness would give the lowest loss at a particular wavelength. This leads to the slight difference in tube thickness value (between different fibers with different geometry) that would give lowest possible loss at any particular wavelength. At $1.55 \mu \mathrm{m}$ the lowest loss in a fiber with 10 cladding tubes is obtained when the tubes have thicknesses of $0.56 \mu \mathrm{m}$ which is also the lowest loss among all the different fibers as illustrated in Fig. 2a.

As the number of ellipses increase, they occupy more area surrounding the core and the loss goes down significantly. To accommodate more ellipses, the sizes of the minor axes need to decrease. However, decreasing the minor axes too much lead to an increase in loss as is evident from Fig. 2a and hence both the number of ellipses and the sizes of minor axes need to be
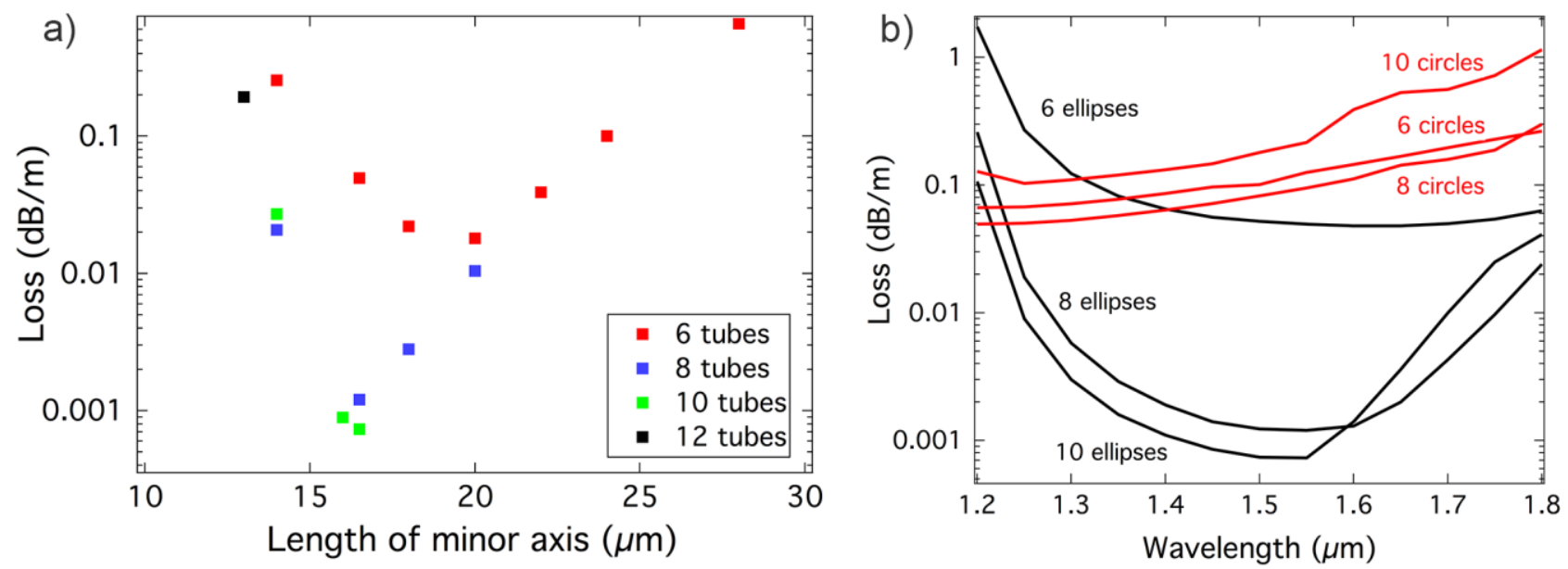

Fig. 2. a) Dependence of loss on the size of minor axis for different number of elliptical tubes in the fiber at a wavelength of $1.55 \mu \mathrm{m}$. The sizes of major axes have been kept constant at $30 \mu \mathrm{m}$. On increasing the minor axes beyond $16.5 \mu \mathrm{m}$ and $13 \mu \mathrm{m}$ for 10 and 12 ellipses respectively, the tubes contact each other. The thicknesses of the tubes have been kept constant at $0.56 \mu \mathrm{m}$ which gives the lowest loss for the fiber with 10 tubes at $1.55 \mu \mathrm{m}$. These thicknesses can be altered to give lower loss for the fiber with lesser cladding tubes but that will still not give lower loss than the fiber with 10 tubes. b) Wavelength dependent loss when the number of elliptical and circular tubes are varied in the fiber. The core diameter, major axes, minor axes and tube thicknesses have been kept constant at 30 $\mu \mathrm{m}, 30 \mu \mathrm{m}, 16.5 \mu \mathrm{m}$ and $0.56 \mu \mathrm{m}$ for all the fibers with elliptical tubes. For the fibers with circular tubes, the core diameters have been fixed at $30 \mu \mathrm{m}$ while the radii of the tubes have been varied accordingly. The thicknesses of the circular tubes have been kept at $0.42 \mu \mathrm{m}$ to give lowest loss at $1.55 \mu \mathrm{m}$ wavelength [16]. 
considered whilst designing the fiber. In Fig. $2 b$ it is shown that a fiber with 10 ellipses (major axes of the ellipses $30 \mu \mathrm{m}$ and the minor axes $16.5 \mu \mathrm{m}$ ) gives the lowest loss. The fiber with 10 ellipses has a loss value at $1.55 \mu \mathrm{m}$ that is 2 orders of magnitude less than the fiber with circular tubes with the same core size [16]. Decreasing the minor axes of the ellipses further would lead to touching cladding tubes, which increases the transmission loss as shown in Fig. 3. However, this increase in transmission loss is not as pronounced as is in the case of a fiber with circular cladding tubes [17]. Regions of contact between the capillary tubes can be considered as additional resonators which increase the overall loss. Due to the increased curvature the fiber with elliptical tubes have less power going into the tubes. Therefore, even if the capillaries come in contact with each other the power in the glass tubes increase by a smaller amount as compared to circular tubes.

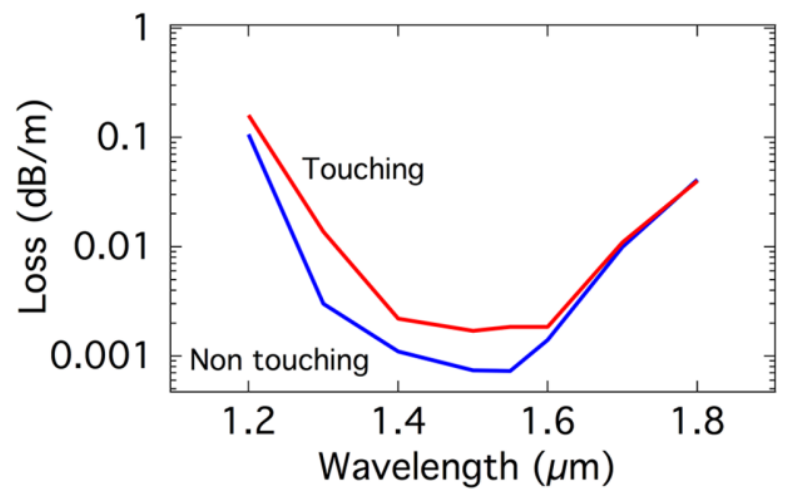

Fig. 3. Wavelength dependent loss for fiber with 10 tubes. When the sizes of minor axes are $16.5 \mu \mathrm{m}$ the tubes are separated, however when they are increased to $17 \mu \mathrm{m}$ they come in contact with each other which increases the loss as shown in the figure. The major axes have been kept constant at $30 \mu \mathrm{m}$.

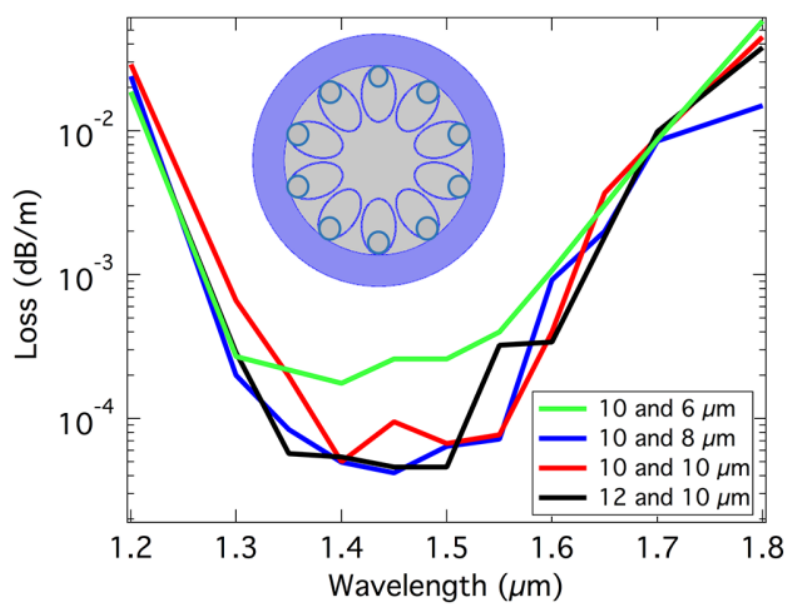

Fig. 4. Wavelength dependent loss when the sizes of the nested tubes are varied. The sizes of the larger outer tubes have been kept constant at $30 \mu \mathrm{m}$ (major axis) and $16.5 \mu \mathrm{m}$ (minor axis). Inset shows the structure of a nested fiber

Recent modeling [16, 19, 26] and subsequent fabrication [20] have shown that adding another circle inside each tube i.e. making nested negative curvature fiber drastically decreases the loss. We have studied the transmission loss for nested elliptical tubes and found a decrease in transmission loss, as shown in Fig. 4. The core size of the fiber has been kept fixed at $30 \mu \mathrm{m}$ diameter. Keeping the outer ellipses with a major axes of $30 \mu \mathrm{m}$ and minor axes of $16.5 \mu \mathrm{m}$, the effect of the sizes of the inner ellipses has been studied. Inner ellipses with axes sizes of 10 $\mu \mathrm{m}$ and $10 \mu \mathrm{m}$ (making them circular) or $10 \mu \mathrm{m}$ and $8 \mu \mathrm{m}$, give almost similar low loss at $1.55 \mu \mathrm{m}$ as shown in Fig. 4 which is much lower than those of nested fibers with circular tubes [16]. Again, touching cladding tubes lead to an increase in transmission loss as shown in Fig. 5. The nested ellipses in the cladding lead to a low loss and a wide transmission window not only in the near infrared, but also in the visible and UV region of the spectrum, as shown in Fig. 6.

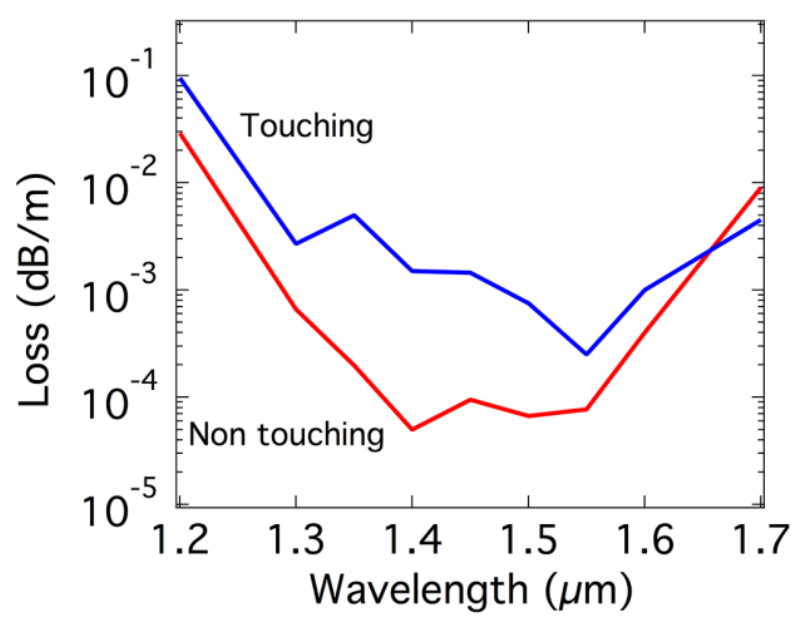

Fig. 5. Wavelength dependent loss showing the increase in loss for a nested fiber when the outer tubes touch each other i.e. when the sizes of minor axes are increased from 16.5 to $17 \mu \mathrm{m}$. Inner tubes have been made circular with diameters of $10 \mu \mathrm{m}$

\section{CONCLUSION}

Negative curvature fibers with elliptical tubes can give low loss guidance over a broad wavelength region. In this paper we have concentrated on getting an efficient design for light guidance at $1.55 \mu \mathrm{m}$ and hence the thicknesses of the tubes were chosen as $0.56 \mu \mathrm{m}$. This tube thickness can be changed to shift the high transmission region to other parts of the spectrum depending on the applications. Along with the tube thickness, other design parameters can also be varied to obtain low loss from UV to IR regions.

The negative curvature fiber has low loss and a much broader transmission bandwidth as compared to photonic bandgap fiber [16]. These features make the negative curvature fiber highly advantageous with tremendous potential applications at wavelengths spanning from UV to mid IR [27-31]. This opens up numerous possibilities for potential applications such as data 


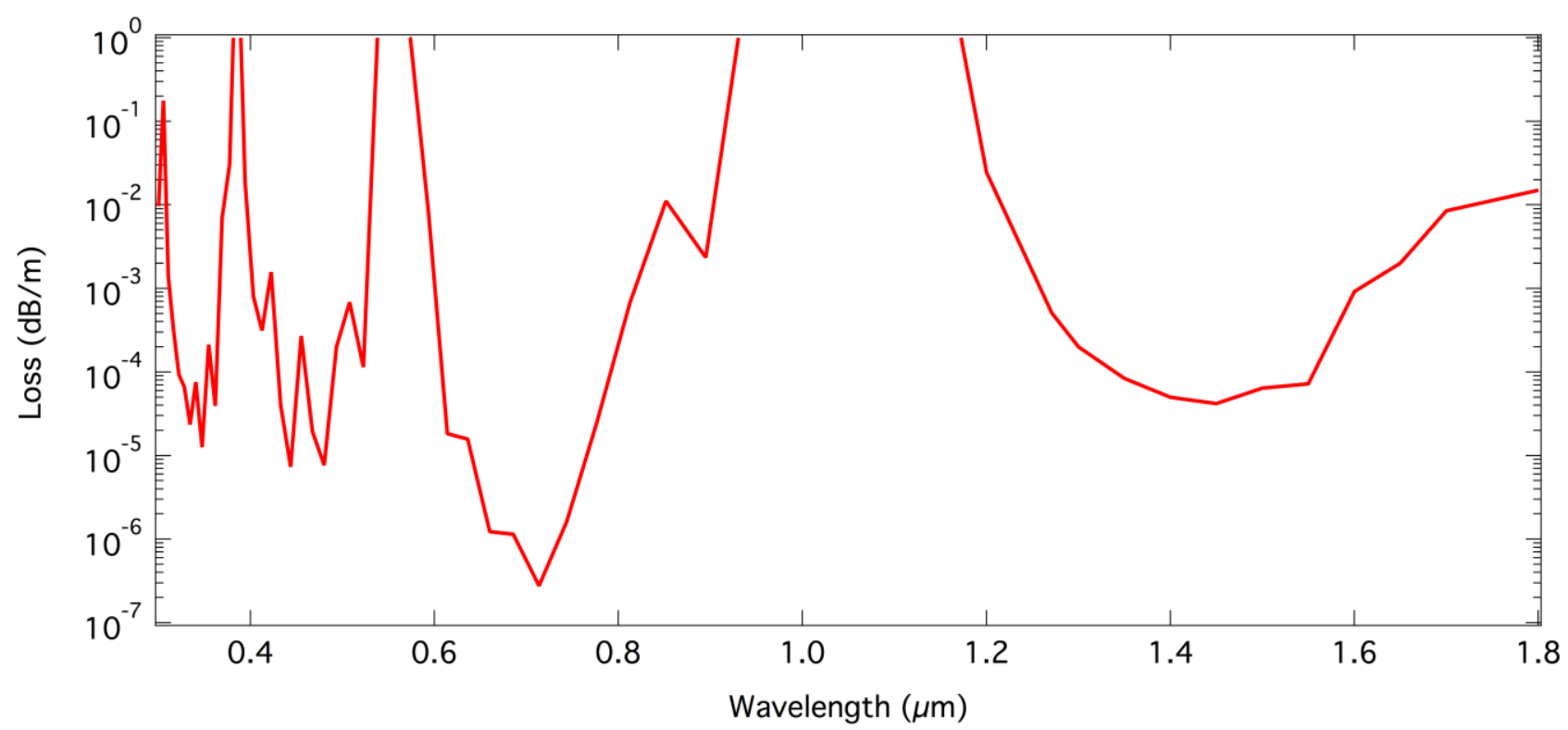

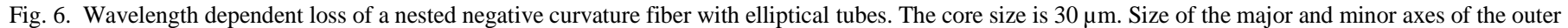
tubes are $30 \mu \mathrm{m}$ and $16.5 \mu \mathrm{m}$ while the inside nested tubes are $10 \mu \mathrm{m}$ and $8 \mu \mathrm{m}$ respectively. Thicknesses of the tubes are $0.56 \mu \mathrm{m}$

transmission, sensing, high power delivery etc. Fused silica [14, 17] and chalcogenide negative curvature fibers [32] with low optical loss in near and mid infrared respectively have already been demonstrated, which can come down further by replacing the circular capillaries with elliptical ones. The fabrication of these fibers with elliptical tubes is challenging, however with extrusion or stack-and-draw, it would be possible to fabricate a preform for this structure. It has been shown that varying the tension during preform fabrication process of fibers with circular cladding tubes, can lead to their elongation [33]. Interplay between the viscosity of glass and tension during the drawing process needs to be studied in order to draw these fibers. The drawing process also has to be conducted carefully to avoid the elliptical elements expanding into circles. However, the benefits of obtaining low loss over such a wide range of wavelengths should encourage further research in this area.

\section{ACKNOWLEDGMENT}

We would like to acknowledge Prof John Badding for helpful suggestions.

\section{REFERENCES}

[1] J. C. Knight, J. Broeng, T. A. Birks, and P. S. J. Russel, "Photonic band gap guidance in optical fibers," Science, vol. 282, pp. 1476-1478, Nov 20 1998.

[2] R. F. Cregan, B. J. Mangan, J. C. Knight, T. A. Birks, P. S. Russell, P. J. Roberts, et al., "Single-mode photonic band gap guidance of light in air," Science, vol. 285, pp. 1537-1539, Sep 31999.

[3] A. Rundquist, C. G. Durfee, Z. H. Chang, C. Herne, S. Backus, M. M. Murnane, et al., "Phase-matched generation of coherent soft X-rays," Science, vol. 280, pp. 1412-1415, May 291998.

[4] M. J. Renn, D. Montgomery, O. Vdovin, D. Z. Anderson, C. E. Wieman, and E. A. Cornell, "Laser-Guided Atoms in Hollow-Core Optical Fibers," Physical Review Letters, vol. 75, pp. 3253-3256, Oct 301995.
[5] M. J. Renn, R. Pastel, and H. J. Lewandowski, "Laser guidance and trapping of mesoscale particles in hollow-core optical fibers," Physical Review Letters, vol. 82, pp. 1574-1577, Feb 151999.

[6] E. A. J. Marcatili and R. A. Schmeltzer, "Hollow Metallic + Dielectric Waveguides for Long Distance Optical Transmission + Lasers," Bell System Technical Journal, vol. 43, pp. 1783-1809, 1964.

[7] J. A. Harrington, "A review of IR transmitting, hollow waveguides," Fiber and Integrated Optics, vol. 19, pp. 211-227, Jul-Sep 2000.

[8] F. Yu, W. J. Wadsworth, and J. C. Knight, "Low loss silica hollow core fibers for 3-4 $\mu \mathrm{m}$ spectral region," Optics Express, vol. 20, pp. 1115311158, May 72012.

[9] A. Hartung, J. Kobelke, A. Schwuchow, J. Bierlich, J. Popp, M. A. Schmidt, et al., "Low-loss single-mode guidance in large-core antiresonant hollow-core fibers," Optics Letters, vol. 40, pp. 3432-3435, July 152015.

[10] P. Uebel, M. C. Günendi, M. H. Frosz, G. Ahmed, N. N. Edavalath, J.-M. Ménard, et al., "Broadband robustly single-mode hollow-core PCF by resonant filtering of higher-order modes," Optics Letters, vol. 41, pp. 1961-1964, May 12016.

[11] B. Temelkuran, S. D. Hart, G. Benoit, J. D. Joannopoulos, and Y. Fink, "Wavelength-scalable hollow optical fibres with large photonic bandgaps for $\mathrm{CO}_{2}$ laser transmission," Nature, vol. 420, pp. 650-653, Dec 122002.

[12] S. Chaudhuri, J. R. Sparks, R. R. He, and J. V. Badding, "Hollow core silicon-silica Bragg fiber," 2015 Conference on Lasers and ElectroOptics (Cleo), 2015.

[13] N. M. Litchinitser, A. K. Abeeluck, C. Headley, and B. J. Eggleton, "Antiresonant reflecting photonic crystal optical waveguides," Optics Letters, vol. 27, pp. 1592-1594, September 152002.

[14] A. D. Pryamikov, A. S. Biriukov, A. F. Kosolapov, V. G. Plotnichenko, S. L. Semjonov, and E. M. Dianov, "Demonstration of a waveguide regime for a silica hollow - core microstructured optical fiber with a negative curvature of the core boundary in the spectral region $>3.5 \mu \mathrm{m}$," Optics Express, vol. 19, pp. 1441-1448, Jan 172011.

[15] F. Yu and J. C. Knight, "Negative Curvature Hollow-Core Optical Fiber," Ieee Journal of Selected Topics in Quantum Electronics, vol. 22, Mar-Apr 2016.

[16] F. Poletti, "Nested antiresonant nodeless hollow core fiber," Optics Express, vol. 22, pp. 23807-23828, Oct 62014.

[17] A. N. Kolyadin, A. F. Kosolapov, A. D. Pryamikov, A. S. Biriukov, V. G. Plotnichenko, and E. M. Dianov, "Light transmission in negative curvature hollow core fiber in extremely high material loss region," Optics Express, vol. 21, pp. 9514-9519, Apr 222013.

[18] W. Belardi and J. C. Knight, "Hollow antiresonant fibers with low bending loss," Optics Express, vol. 22, pp. 10091-10096, April 212014. 
[19] W. Belardi and J. C. Knight, "Hollow antiresonant fibers with reduced attenuation," Optics Letters, vol. 39, pp. 1853-1856, Apr 12014.

[20] W. Belardi, "Design and Properties of Hollow Antiresonant Fibers for the Visible and Near Infrared Spectral Range," Journal of Lightwave Technology, vol. 33, pp. 4497-4503, Nov 12015.

[21] M. S. Habib, O. Bang, and M. Bache, "Low-loss single-mode hollow-core fiber with anisotropic anti-resonant elements," Optics Express, vol. 24, pp. 8429-8436, April 182016.

[22] M. S. Habib, O. Bang, and M. Bache, "Anisotropic Anti-resonant Elements gives Broadband Single-mode Low-loss Hollow-core Fibers," in Conference on Lasers and Electro-Optics, San Jose, California, 2016, p. JTu5A.99.

[23] Y. Y. Wang, N. V. Wheeler, F. Couny, P. J. Roberts, and F. Benabid, "Low loss broadband transmission in hypocycloid-core Kagome hollowcore photonic crystal fiber," Optics Letters, vol. 36, pp. 669-671, March 12011.

[24] L. Vincetti and V. Setti, "Waveguiding mechanism in tube lattice fibers," Optics Express, vol. 18, pp. 23133-23146, October 252010.

[25] W. Belardi and J. C. Knight, "Effect of core boundary curvature on the confinement losses of hollow antiresonant fibers," Optics Express, vol. 21, pp. 21912-21917, Sep 232013.

[26] M. S. Habib, O. Bang, and M. Bache, "Low-loss hollow-core silica fibers with adjacent nested anti-resonant tubes," Optics Express, vol. 23, pp. 17394-17406, Jun 292015.

[27] P. Jaworski, F. Yu, R. R. J. Maier, W. J. Wadsworth, J. C. Knight, J. D. Shephard, et al., "Picosecond and nanosecond pulse delivery through a hollow-core Negative Curvature Fiber for micro-machining applications," Optics Express, vol. 21, pp. 22742-22753, Sep 232013.

[28] P. Jaworski, F. Yu, R. R. J. Maier, W. J. Wadsworth, T. A. Birks, J. C. Knight, et al., "Delivery of high-power nanosecond and picosecond pulses through a hollow-core Negative Curvature Fibre for micro-machining applications," 2013 Conference on and International Quantum Electronics Conference Lasers and Electro-Optics Europe (Cleo Europe/Iqec), 2013.

[29] P. Jaworski, F. Yu, R. M. Carter, J. C. Knight, J. D. Shephard, and D. P. Hand, "High energy green nanosecond and picosecond pulse delivery through a negative curvature fiber for precision micro-machining," Optics Express, vol. 23, pp. 8498-8506, Apr 62015.

[30] D. V. Bogdanovich, A. Shristava, V. G. Chigrinov, A. S. Biriukov, and A. D. Pryamikov, "Hollow core negative curvature fibre with layers of photoaligned optically anisotropic material," Laser Physics Letters, vol. 12, Oct 2015.

[31] S. N. Turtaev, M. I. Belovolov, A. E. Levchenko, A. F. Kosolapov, A. D. Pryamikov, and A. N. Kolyadin, "Acoustic Sensitivity of the Negative Curvature Hollow Core Fiber," 2014 International Conference Laser Optics, 2014.

[32] A. F. Kosolapov, A. D. Pryamikov, A. S. Biriukov, V. S. Shiryaev, M. S. Astapovich, G. E. Snopatin, et al., "Demonstration of $\mathrm{CO}_{2}$-laser power delivery through chalcogenide-glass fiber with negative-curvature hollow core," Optics Express, vol. 19, pp. 25723-25728, Dec 52011.

[33] A. F. Kosolapov, G. K. Alagashev, A. N. Kolyadin, A. D. Pryamikov, A. S. Biryukov, I. A. Bufetov, et al., "Hollow-core revolver fibre with a double-capillary reflective cladding," Quantum Electronics, vol. 46, p. 267, 2016. 